\title{
The prognosis of out-patient alcohol treatment among parents with childcare responsibility
}

\author{
Angelina Isabella Mellentin, Annette Elkjær Ellermann, Bent Nielsen, Anna Mejldal, Sören Möller and \\ Anette Søgaard Nielsen
}

\section{Background}

Despite expansive knowledge on the detrimental effects of growing up with parents with alcohol use disorders (AUDs), little is known about the prognosis of alcohol treatment among parents with childcare responsibility.

\section{Aims}

This observational cohort study aimed to examine the prognosis of patients with and without childcare responsibility, in a conventional out-patient alcohol treatment clinic.

\section{Method}

A consecutive AUD sample ( $N=2201)$, based on ICD-10 Diagnostic Criteria for Research, was assessed with the European Addiction Severity Index during the clinical routine, at treatment entry and conclusion. Data on addiction severity, treatment course and drinking outcomes were derived, and adjusted odds ratios (AORs) were calculated with logisticregression models. Drinking outcomes were compared in an intention-to-treat analysis, including all patients in a logistic regression with inverse probability weighting.

\section{Results}

Patients with childcare responsibility (aged $<18$ years) had a less severe addiction profile and lower drop-out rate compared with patients without children or with children living out-of-home. They were also more likely to improve on all drinking-related outcomes, including abstinence (AOR 2.68, 95\% Cl 1.82-3.95), number of drinking days (AOR $2.45,95 \% \mathrm{Cl} 1.50-4.03$ ) and excessive drinking days (AOR 4.66, 95\% $\mathrm{Cl} 2.36-9.17$ ); and those with children living out-of-home had better outcomes on abstinence (AOR 1.59, 95\% Cl 1.08-2.34) than patients without children.

\section{Conclusions}

Childcare responsibility among out-patients was associated with better treatment course and outcomes than those without or not living with their children. This knowledge can help guide clinical practice, effectuate interventions and inform social authorities.

\section{Keywords}

Alcohol disorders; out-patient treatment; outcome studies; childhood experience.

\section{Copyright and usage}

(C) The Royal College of Psychiatrists 2018. This is an Open Access article, distributed under the terms of the Creative Commons Attribution-NonCommercial-ShareAlike licence (http://creativecommons.org/licenses/by-nc-sa/4.0/), which permits non-commercial re-use, distribution, and reproduction in any medium, provided the same Creative Commons licence is included and the original work is properly cited. The written permission of Cambridge University Press must be obtained for commercial re-use.

\section{Background}

Previous studies have focused extensively on children who are exposed to parental alcohol use disorder (AUD). It has been estimated that as many as $22-30 \%$ of the children in the general population are exposed to parental AUD, ${ }^{1-3}$ and evidence indicates their high risk of exposure to adverse experiences, including chronic family stress, ${ }^{4,5}$ disharmony and conflicts in the family, ${ }^{6,7}$ inter-parental violence ${ }^{8,9}$ and emotional, physical and sexual child abuse, ${ }^{10-12}$ which can lead to family separation and foster care. ${ }^{12}$ Along with genetic susceptibility, adverse childhood experiences may manifest as psychiatric disorders later in life, and it is particularly well established that these children are at a greater risk of developing AUD and other substance use disorders (SUDs). ${ }^{2,8,13-16}$

It is imperative to intervene against negative intergenerational heritage, and once a parent has developed an AUD, the best way to intervene is by treating the parent(s) for their AUD.

Improving child welfare is best attained by enhancing the function within the family, by offering the parents supportive initiatives and relevant treatment, as placement in foster care risks another adverse experience. ${ }^{17-22}$ However, there is little knowledge pertaining to the association between childcare responsibility and outcomes of treating parents for AUD. It has been found that parents with AUD are motivated to be treated, to relieve their children of the negative effects of the AUD and avoid intervention from social authorities (i.e. monitoring or forced removal of the children to foster care). ${ }^{8,23,24}$ Further, a small-scale study conducted in $1991(n=130)$ found that treatment-seeking patients with AUD, with a relatively low degree of addiction impairment and who were living with their children, were less likely to drop out, more likely to comply and achieved better drinking-related outcomes compared with patients without similar childcare responsibility. ${ }^{25}$ Other studies found that parents with other types of SUD, who had children living with them, achieved similar or even better drug-related outcomes compared with parents without childcare responsibility. ${ }^{26-28}$ However, other adverse psychosocial circumstances could have affected the overall severity of the addiction of parents with other SUDs of mainly illegal drugs, such as heroin, cocaine and poly-substances. Neither the early study on AUD nor the majority of later SUD studies investigated the effect of gender on treatment outcomes, but it is plausible that living with children may affect women more than men, as they often have the primary childcare responsibility. ${ }^{26,27}$

A contemporary and large-scale study pertaining to the specific prognosis of treating AUDs, as well as its associations with parental gender, is warranted to guide clinical practice, effectuate interventions and inform social authorities within this field.

\section{Aim}

This study aimed to investigate whether childcare responsibility (living with children aged $<18$ years) is related to the addiction severity profile at treatment entry, the treatment course and drinking-related outcomes, when treated in conventional evidence-based pharmacological and psychosocial out-patient AUD treatment. Based on the findings from the early AUD study together with 
the SUD studies mentioned above, we hypothesized that in parents with AUD, living with children is associated with a better treatment prognosis relative to patients without children and parents without childcare responsibilities.

\section{Method}

This observational study is reported in accordance with the Reporting of Studies Conducted using Observational RoutinelyCollected Health Data (RECORD) guidelines, ${ }^{29}$ an extension of the Strengthening the Reporting of Observational Studies in Epidemiology (STROBE) guidelines. ${ }^{30}$ These guidelines were applied to ensure an adequate reporting of the study.

The authors assert that all procedures contributing to this work comply with the ethical standards of the relevant national and institutional committees on human experimentation and with the Helsinki Declaration of 1975, as revised in 2008. Formal ethical approval was not required for this study, in accordance with Danish legislation; however, the use of data from the database for research purposes was approved by the Danish Data Protection Agency (region of Southern Denmark, registration number: 2012-58-0018).

\section{Design and setting}

The study was conducted as an observational cohort study in an out-patient alcohol treatment clinic located in Odense, Denmark. AUD is usually treated at out-patient municipal treatment facilities and only alcohol-related problems are addressed there. Public outpatient treatment is free of charge to patients, open for self-referral and anonymity is available. If patients suffer from either illegal SUDs, have severe psychiatric comorbidity (e.g. acute psychotic disorders), severe cognitive impairment (e.g. dementia) or terminal somatic illness, treatment takes place elsewhere.

\section{Treatment}

The clinic cooperates closely with the Odense University Hospital and the Unit of Clinical Alcohol Research, University of Southern Denmark. The pharmacological and psychosocial treatment offered at the clinic is evidence-based and provided in accordance with the national clinical recommendations. ${ }^{31,32}$ Upon entering treatment, patients were provided a medical consultation by a psychiatrist, and other pharmacological treatment targeting the AUD and detoxification was provided as was deemed necessary. Detoxification was conducted with benzodiazepines and disulfiram, acamprosate and/or naltrexone were also prescribed when appropriate. After detoxification, psychiatrists referred the patients to psychosocial treatment, which constituted the main component of the offered treatment and was carried out by the team of therapists in the clinic, comprising nurses and social workers trained within the treatment range. Treatment began with a motivating interview, to engage the patients in their treatment, followed by cognitivebehavioural therapy or supportive consultations focused on the AUD. All treatments were carried out during $1 \mathrm{~h}$ individual or group sessions. At the beginning of the treatment, sessions were offered once a week, decreasing to twice a month after 4 weeks. A treatment course was scheduled to last 3 months and comprised eight psychosocial sessions, after which an evaluation of the treatment course was conducted. The duration of treatment was decided together with the patient on an individual basis, and could be extended as long as needed. Frequent supervision of the staff was undertaken, and psychiatrists monitored the treatment course regularly.

\section{Participants}

The population consisted of a consecutive sample of patients with harmful use or dependence of alcohol (AUD), entering the outpatient alcohol treatment clinic from October 2006 to October 2016. To be eligible for the study, patients had to have an AUD diagnosis; be aged between 18 and 65 years to assure that they may have children $<18$ years of age; provide information about biological or adopted children, and the age of the youngest child; have at least one child $<18$ years of age if having children, to assure that not all the children were adults and finally, have finalised or dropped out of the treatment course (i.e. not currently in treatment). A total of 3569 patients entered treatment and were discharged during this period, of which 1368 were excluded from the study for following reasons: 82 patients did not fulfil the criteria for an AUD, 121 patients were not aged between 18 and 65 years, eight patients did not provide information concerning children in the household, 1049 patients only had grown-up children aged $\geq 18$ years and finally, 186 patients were still/currently in treatment when data were collected/when the study period concluded. Hence, 2201 patients were eligible and included in the study.

\section{Assessment}

Data was obtained from the clinical database in the treatment clinic, developed with the purpose of monitoring the overall quality of treatment and conducting research to improve the treatment. All patients entering treatment are required to complete a baseline assessment interview, after medical consultation and before referral to psychosocial treatment, and a follow-up assessment every 3 months and upon treatment conclusion. The clinical data are stored in the database, and data derived for further analysis are encrypted and no person sensitive information is extracted.

The baseline assessment was performed using the ICD-10 Diagnostic Criteria for Research for harmful use of alcohol (F10.1) and alcohol dependence syndrome (F10.2), ${ }^{29,30}$ and the European Addiction Severity Index (EuropASI) was applied both at baseline and follow-up. ${ }^{31-33}$ The EuropASI assesses sociodemographic, clinical AUD-related variables (e.g. years with AUD, prior treatment etc.) and degree of addiction severity related to nine areas of the patients life, including alcohol use and other drug use, medical and psychiatric status, family status and other social status, economy and job satisfaction, and legal status. Further, for each area, a composite score ranging from 0 to 1 was calculated, with higher scores reflecting higher severity of problems. These composite scores are exclusively derived from items relating to the past 30 days. ${ }^{32,34}$

All assessments were performed in face-to-face interviews by the team of therapists in the clinic, who were trained in the assessment procedures.

\section{Measures}

The baseline sample characteristics were derived from ICD-10 and baseline EuropASI assessment, and variables related to treatment course and outcomes were based on baseline and latest follow-up EuropASI assessment, i.e. the treatment conclusion.

The sample was divided into three patient groups: group A, comprising patients with no children; group $\mathrm{B}$, comprising patients with at least one child $<18$ years of age (biological or adopted) living at home; and group $\mathrm{C}$, comprising patients with at least one child $<18$ years of age (biological or adopted), living out-of-home.

To describe sample characteristics, the following sociodemographic, clinical AUD-related variables and degree of addiction severity were calculated: age, gender, cohabitation (married or living with a partner), cohabiting with a partner with AUD, vocational education (education leading to a profession, e.g. vocational 
training, a bachelor's degree at vocational academies or university colleges ( $\leq 4$ years of education), or a university degree or other higher education ( $>4$ years of education) after finishing elementary or high school), employment (full- or part-time employment for the past 3 years), AUD diagnoses (harmful use or dependence), delirium tremens (one or more episodes during lifetime), years with AUD, prior AUD treatment (in-patient or out-patient treatment), referral form (self-referred or referred by the social services), drinking measures (i.e. abstinence, drinking days and days with excessive drinking the past 30 days) and the nine EuropASI composite scores (also based on items relating to the past 30 days).

The treatment course variables, consisting of whether patients concluded treatment successfully or dropped out, were based on the latest follow-up assessment, and outcome measures were derived from the EuropASI's alcohol use component, comprising abstinence, drinking days and excessive drinking days. Abstinence was defined as not consuming any alcohol, a drinking day as consuming minimum one unit of alcohol (12 g pure ethanol) and days with excessive drinking as drinking six or more units of alcohol in the 30 days preassessment. Abstinence outcomes were calculated from the treatment conclusion, indicating continued (from baseline) or acquired abstinence during treatment. Drinking days and days with excessive drinking were defined as change scores from baseline to treatment conclusion, indicating whether a decrease or increase occurred in these measures.

\section{Statistical analyses}

The range and distribution of sociodemographic, AUD-related and addiction severity variables at baseline and the treatment course were examined by $\chi^{2}$ tests for categorical data, and Kruskal-Wallis rank-sum tests for continuous data. To examine whether the total sample exhibited an improvement from baseline to follow-up, McNemar's $\chi^{2}$ tests and Wilcoxon signed-rank tests were conducted on categorical and continuous drinking outcomes, respectively.

An intention-to-treat analysis approach was adopted for all treatment outcomes and was analysed by weighted logistic regression, to examine whether the groups differed in their achievements. Further, the logistic regression models were adjusted for the sociodemographic, AUD-related and addiction severity variables that were found to differ between groups at baseline, and were stratified by gender. Inverse probability weighting (IPW), which addresses the potential bias arising from the exclusion of patients with missing data at follow-up, ${ }^{35}$ provided weights in the model. The IWP method weights each observation by the inverse of the probability of having completed a follow-up, to create a weighted subsample of complete cases that resembled the full sample, with respect to chosen observed predictors. The IPW weights were constructed with a logistic model to predict the probability of having answered the question of alcohol consumption in the 30 days before follow-up. The IPW model contained the following predictor variables: age, gender, education, employment, cohabitation, referral form, previous AUD treatment, years of AUD, delirium tremens and psychiatric and somatic composite scores at baseline. As $6 \%$ of the patients $(n=206)$ had missing data on these baseline variables and thus did not receive a weight, a sensitivity analysis was performed, giving these cases a weight of 1 . All analyses were conducted with Stata v15 for Windows.

\section{Results}

\section{Sample characteristics}

At baseline, $41 \%$ of the patients ( $n=900$ ) belonged to group A, 34\% $(n=746)$ belonged to group B and 25\% $(n=550)$ belonged to group
C (Table 1). Group A had the lowest median age, and group B (39\%) comprised more female patients than group A (21\%) and group C (18\%). Group B was also more likely to be cohabiting, have completed their education and be employed, compared with the other groups. Further, group B patients had less severe AUD, fewer years of AUD, were less likely to have suffered from delirium tremens and were less likely to have been in treatment before, whereas group $\mathrm{C}$ demonstrated the opposite, with the most severe AUD patterns among this group. Group B patients were also more prone to seek treatment on their own or the family's initiative, whereas group $\mathrm{C}$ was more likely to be referred to treatment by social authorities, followed by group B patients. When the patients are referred by social services, the treatment is not mandated, but the patients may feel forced to complete the treatment as there is awareness of their issues that could ultimately have consequences for child custody if they do not resolve their drinking problem.

At baseline drinking measures, the groups only differed on drinking days: group B posted the lowest and group C posted the highest number of drinking days. Regarding the addiction severity reflected by the composite scores, group B was the least impaired on alcohol use, medical status and economy, but the most impaired on family status. Groups A and C were similarly impaired on alcohol use, medical status and economy, but group $\mathrm{C}$ was (second to group B) the most impaired on family status and group A was the most impaired on social status.

In general, all patients functioned relatively well, as is apparent from the low composite scores of the groups; impairment was reflected mainly in the alcohol use composite score.

\section{Treatment course and outcomes}

More than half of all patients (55\%) successfully concluded the treatment course (45\% drop-out rate). Among those that concluded treatment, $60 \%$ were abstinent at follow-up, which was a significant improvement from the baseline abstinence rate (McNemar's $\chi^{2}=$ $406.16, P<0.001)$. In total, $89 \%$ had a decline in number of drinking days, from a median of 15 (interquartile range [IQR] 25) at baseline to 0 (IQR 3) at follow-up (Wilcoxon signed-rank test $z=24.51$, $P<0.001$ ), and $94 \%$ had a decline in number of excessive drinking days, decreasing from a median of 14 (IQR 25) to 0 (IQR 0) (Wilcoxon signed-rank test $z=25.68, P<0.001$ ). Thus, an overall significant improvement was observed in all drinking outcomes, from baseline to follow-up, regardless of group assignment. Group B was the most treatment compliant, with $63 \%$ successfully concluding treatment and only $37 \%$ prematurely dropping out, whereas in groups A and B, 51 and $50 \%$ successfully concluded treatment and 49 and $50 \%$ dropped out, respectively.

Treatment outcomes are presented in Table 2 as odds ratios and adjusted odds ratios (AORs), and the models were adjusted for age, gender, cohabitation, education, employment, referral form, AUD diagnosis, delirium tremens, years with AUD and relevant composite scores, as these variables were not equally distributed at baseline. Better outcomes were observed in group B, where the odds ratios and AORs of achieving a positive alcohol-related outcome on all parameters were highly significant in relation to group A. Odds ratios for group $\mathrm{C}$ also improved, compared with group $\mathrm{A}$, in continued or acquired abstinence as well as in the decline in number of excessive drinking days from baseline to follow-up, but only the former remained significant in AORs. Treatment outcomes stratified by gender are presented in Table 3 as odds ratios and AORs, where the models are adjusted for the same variables as the former inferential analysis presented in Table 2, except for gender. When the treatment outcomes were stratified by gender, even better outcomes were observed in group B among women, 
Table 1 Baseline characteristics of treatment-seeking patients with alcohol use disorder (AUD) in an operating Danish alcohol treatment clinic ( $N=2201)$

\begin{tabular}{|c|c|c|c|}
\hline & $\begin{array}{l}\text { Group A: no children, } \\
\qquad n=900\end{array}$ & $\begin{array}{l}\text { Group B: children living at home, } \\
\qquad n=746\end{array}$ & $\begin{array}{l}\text { Group C: Children living out-of-home, } \\
\qquad n=555\end{array}$ \\
\hline \multicolumn{4}{|l|}{ Demographics } \\
\hline Age (median, IQR)* & $39(19)$ & $42(9)$ & $42(9)$ \\
\hline Age range & $18-64$ & 19-61 & $22-62$ \\
\hline Female, $n(\%)^{*}$ & $187(21)$ & 288 (39) & $102(18)$ \\
\hline Cohabitation, $n(\%)^{\mathrm{a} \star}$ & $263(29)$ & $519(70)$ & $119(21)$ \\
\hline Cohabiting with a partner with AUD, $n(\%)^{\text {a }}$ & $64(7)$ & $46(6)$ & $24(4)$ \\
\hline Completed education, $n(\%)^{\mathrm{b*}}$ & 577 (64) & $560(75)$ & $354(64)$ \\
\hline Employment, $n(\%)^{\mathrm{c} *}$ & $385(43)$ & $469(63)$ & $266(48)$ \\
\hline \multicolumn{4}{|l|}{ AUD severity, prior treatment and referral form } \\
\hline \multicolumn{4}{|l|}{$A U D^{*}$} \\
\hline Harmful use of alcohol, $n$ (\%) & $138(15)$ & $172(23)$ & $64(12)$ \\
\hline Alcohol dependency syndrome, $n$ (\%) & $762(85)$ & $574(77)$ & $491(88)$ \\
\hline Delirium tremens, $n(\%)^{d_{\star}}$ & $102(11)$ & $45(6)$ & $72(13)$ \\
\hline Years with AUD (median, IQR)* & $11(14)$ & $10(14)$ & $13(14)$ \\
\hline Previous AUD treatment, $n(\%)^{*}$ & $423(47)$ & 308 (41) & $285(51)$ \\
\hline Self-referred (on own or family's initiative), $n(\%)^{*}$ & $410(46)$ & $416(56)$ & $251(45)$ \\
\hline Referred to treatment by social services, $n(\%)^{*}$ & $51(6)$ & $72(10)$ & $71(13)$ \\
\hline \multicolumn{4}{|l|}{ Drinking measures ${ }^{\mathrm{e}}$} \\
\hline Abstinence, $n(\%)$ & $120(13)$ & $96(13)$ & 85 (15) \\
\hline Drinking days (median/IQR)* & $17(24)$ & $15(25)$ & $20(24)$ \\
\hline Excessive drinking days (median/IQR) & $15(25)$ & $14(23)$ & $15(24)$ \\
\hline \multicolumn{4}{|l|}{ Addiction Severity Index, composite scores (median, IQR) } \\
\hline Alcohol use* & $0.68(0.34)$ & $0.64(0.34)$ & $0.68(0.34)$ \\
\hline Drug use & $0(0.27)$ & $0(0.52)$ & $0(0.23)$ \\
\hline Medical status* & $0.25(0.67)$ & $0.11(0.58)$ & $0.24(0.67)$ \\
\hline Psychiatric status & $0.18(0.39)$ & $0.16(0.36)$ & $0.18(0.41)$ \\
\hline \multicolumn{4}{|l|}{ Social status } \\
\hline Family status* & $0(0.025)$ & $0(0.47)$ & $0(0.35)$ \\
\hline Other social status* & $0(0)$ & $0(0)$ & $0(0)$ \\
\hline \multicolumn{4}{|l|}{ Employment } \\
\hline Economy* & $1(1)$ & $0(1)$ & $1(1)$ \\
\hline Job satisfaction & $0.17(0.67)$ & $0.19(0.67)$ & $0.25(0.67)$ \\
\hline Legal status & $0(0.2)$ & $0(0.2)$ & $0(0.2)$ \\
\hline
\end{tabular}

Table 2 Treatment outcomes among treatment seeking patients in an operating Danish alcohol treatment institution, by having and/or living with children, or not

\begin{tabular}{|c|c|c|c|}
\hline & No children & Children living at home & Children living out-of-home \\
\hline Outcomes & Reference & \multicolumn{2}{|c|}{ Crude odds ratios, $95 \% \mathrm{Cls}$} \\
\hline Continued or acquired abstinence $\mathrm{a}^{\mathrm{a}, \mathrm{c}}$ & 1 & $2.49(1.77-3.51)^{\star * *}$ & $1.76(1.21-2.57)^{\star * *}$ \\
\hline Decline in days with drinking ${ }^{\mathrm{b}, \mathrm{c}}$ & 1 & $2.07(1.31-3.25)^{\star *}$ & $1.15(0.72-1.86)$ \\
\hline Decline in days with excessive drinking ${ }^{b, c}$ & 1 & $4.34(2.23-8.43)^{\star \star *}$ & $1.88(1.01-3.50)^{*}$ \\
\hline Outcomes & Reference & \multicolumn{2}{|c|}{ Adjusted odds ratios, $95 \%$ Cis } \\
\hline Continued or acquired abstinence $e^{a, c}$ & 1 & $2.68(1.82-3.95)^{\star * *}$ & $1.59(1.08-2.34)^{\star}$ \\
\hline Decline in days with drinking ${ }^{\mathrm{b}, \mathrm{c}}$ & 1 & $2.46(1.50-4.03)^{\star \star * *}$ & $1.17(0.71-1.92)$ \\
\hline Decline in days with excessive drinking ${ }^{b, c}$ & 1 & $4.66(2.36-9.17)^{\star \star \star}$ & $1.85(0.97-3.53)$ \\
\hline \multicolumn{4}{|c|}{$\begin{array}{l}\text { a Variable from the last follow-up interview, indicating continued/acquired abstinence or drinking every day at follow-up; }{ }^{b} \text { change score from baseline to the last follow-up, indicating a } \\
\text { decline in alcohol consumption; ' inverse probability weighting applied. } \\
* P<0.05, * * P<0.01, * * * P<0.001 \text {. }\end{array}$} \\
\hline
\end{tabular}

whereas the small significant effect in group $\mathrm{C}$ disappeared after gender stratification.

\section{Discussion}

In patients with AUD who are seeking treatment, those living with children showed a less severe addiction profile at treatment entry, were more compliant and had a lower drop-out rate during the treatment course compared with their counterparts with no children or children living out-of-home. Strong positive associations were found between living with children and all drinking outcomes, and gender stratification revealed that females achieved the most favourable outcomes. In addition, a positive, although less strong, association was found on abstinence outcome among parents not living with children, but gender stratification resulted in non-significant outcomes among both genders. The best outcomes were found among patients living with their children.

Our findings support the previous conclusion of a small-scale study that found having childcare responsibility was positively associated with a parent's prognosis in AUD treatment. ${ }^{25}$ Although the previous study's sample was derived from an in-patient setting compared with this study's out-patient one, it similarly reported a relatively low degree of addiction severity among parents with AUD with children, and a minor degree of psychosocial impairment among those living with their children, indicating that children are a protective factor in the prevention of a severe addiction pattern. The previous study also had a lower drop-out rate and 


\begin{tabular}{|c|c|c|c|}
\hline Outcomes & No children & Children living at home & Children living out-of-home \\
\hline Women & Reference & \multicolumn{2}{|c|}{ Crude odds ratios, $95 \% \mathrm{Cls}$} \\
\hline Continued or acquired abstinence & 1 & $2.18(1.28-3.73)^{\star *}$ & $1.99(0.89-4.44)$ \\
\hline Decline in days with drinking & 1 & $5.43(2.12-13.87)^{* * *}$ & $0.77(0.30-2.03)$ \\
\hline \multirow[t]{2}{*}{ Decline in days with excessive drinking } & 1 & $6.63(2.02-21-81)^{\star *}$ & $1.54(0.40-5.96)$ \\
\hline & Reference & \multicolumn{2}{|c|}{ Adjusted odds ratios, $95 \% \mathrm{Cls}$} \\
\hline Continued or acquired abstinence & 1 & $2.21(1.23-3.99)^{* *}$ & $2.00(0.90-4.48)$ \\
\hline Decline in days with drinking & 1 & $5.50(1.94-15.58)^{\star * *}$ & $0.76(0.26-2.25)$ \\
\hline Decline in days with excessive drinking & 1 & $6.54(1.91-22.25)^{\star *}$ & $1.82(0.43-7.80)$ \\
\hline Men & Reference & \multicolumn{2}{|c|}{ Crude odds ratios, $95 \% \mathrm{Cls}$} \\
\hline Continued or acquired abstinence & 1 & $1.08(0.78-1.49)$ & $1.32(0.93-1.88)$ \\
\hline Decline in days with drinking & 1 & $1.40(0.83-2.35)$ & $1.23(0.71-2.14)$ \\
\hline \multirow[t]{2}{*}{ Decline in days with excessive drinking } & 1 & $3.61(0.61-8.09)^{* *}$ & $1.92(0.95-3.88)$ \\
\hline & Reference & \multicolumn{2}{|c|}{ Adjusted odds ratios, $95 \% \mathrm{Cls}$} \\
\hline Continued or acquired abstinence & 1 & $1.31(0.89-1.93)$ & $1.30(0.90-1.87)$ \\
\hline Decline in days with drinking & 1 & $1.83(1.04-3.24)^{*}$ & $1.26(0.71-2.23)$ \\
\hline Decline in days with excessive drinking & 1 & $4.08(1.79-9.30)^{* * *}$ & $1.88(0.91-3.85)$ \\
\hline
\end{tabular}

higher compliance during the treatment course, and drinkingrelated outcomes were more positive at 6- and 12-month followup after treatment initiation. Additionally, living with children was found to be the strongest predictor of recovery out of 80 background variables, such as age, gender, education, employment, and non-alcoholic family background. This study's findings reflect similar conclusions in the contemporary context, and add to prior knowledge with the finding that the protective influence of children may be most prominent among female parents with AUD.

Our findings were also consistent with observations of prior studies suggesting that those with SUD that have childcare responsibilities improves treatment outcomes for legal and illegal drugs. ${ }^{26-28}$ Stewart et al's study of 1075 treatment-seeking individuals with SUD in out-patient and in-patient treatments for misuse or dependence of illegal substances in England found that, despite their more severe addiction profiles (most were women with children in their care), their consumption of illicit drugs was reduced at 12-month follow-up after treatment initiation, although not significantly more for patients living with children compared with patients not living with their children, and no difference was found between parental gender. ${ }^{26}$ Comiskey and colleagues examined 404 individuals with SUD (23\% had children in their care) in both clinical settings in Ireland, with more severe addictionrelated problems. ${ }^{27,36}$ In contrast to the findings of Stewart et al, they found that significantly fewer individuals with children in their care were using drugs at 1 -year follow-up, ${ }^{27}$ and the effect persisted after 3 years. ${ }^{28}$ However, the study did not examine parental gender effects, but these findings are in line with gender-specific studies on mothers with SUD, suggesting that increased retention rates and better outcomes were found among women whose children joined them during residential treatment. ${ }^{37-40}$

As this study focused only on patients with AUD, it can be assumed that they were relatively less psychosocially impaired compared with the individuals in other studies, who suffered mainly from heroin or other opiate addiction as well as poly-substance addictions. However, patients with AUD as their only or at least primary diagnosis may also have a severe addiction profile. ${ }^{41-43}$ Therefore, the treatment of parents with AUD with a pronounced severe addiction profile may, to a higher degree, benefit from being enrolled in a broader and complex integrative treatment, where several other supportive and treatment initiatives are effectuated, including parenting skills training and provision of support to children and other family members. ${ }^{44-46}$ Furthermore, we do not know whether the patients included in this study could have achieved even better treatment outcomes if the out-patient treatment was combined with other initiatives.

Women with childcare responsibilities have also been found to be reluctant toward seeking treatment because of fear that social authorities could intervene by taking their children from their care. ${ }^{47,48}$ Although this issue was not examined directly in this study, it was observed that more women with children attended treatment compared with their male counterparts, and that patients living with their children seek treatment at a relatively earlier stage of AUD compared with those who do not live with their children. Additionally, we observed a relatively higher proportion of patients referred by social services and living with their children, indicating that social services encourage and support families in solving the drinking problem. As AUD negatively affects children and increases the risk of a series of psychiatric disorders, including SUDs, ${ }^{2,8,13-16}$ collaboration between social services and treatment for addiction plays an important role in ending the negative intergenerational heritage of alcohol use and dependence within the family.

\section{Strengths and limitations}

The primary strength of this study is the use of a large sample of patients with AUD in an operating out-patient alcohol treatment clinic.

A number of limitations can be mentioned. First, although we adjusted the models with non-comparable baseline sample characteristics across the groups, the fact that the group living with children was less impaired may have affected the results. Prior studies have demonstrated that lower addiction severity at baseline is associated with better treatment outcome. ${ }^{49}$ However, lower impairment at treatment entry may also reflect the protective role of children in the home. Second, the data were self-reported. Although they are generally considered reliable and valid, they may be less so when children are involved because of fear of reprisals from the social authorities. Third, the treatment outcome measures were based on the assessment of the past 30-day baseline and followup. Hence, vital information that occurred in-between this period could have been overlooked. As the outcome data violated assumptions for regression models with continuous outcomes, the 
outcomes were coded as binary variables, and much information might have been lost in the translation procedure. Moreover, the follow-up window failed to consider the possibility that certain patients might have developed a negative drinking outcome after follow-up, and could potentially develop negative treatment outcomes in the longer term. Fourth, although this study was conducted in a naturalistic setting, giving an optimal setting for the generalisability of the study, all patients functioned relatively well, as is apparent from the low composite scores in the three study groups, where the impairment was reflected mainly in the alcohol use composite score. Thus, this might compromise the external validity of the study as the sample could be representative of patients with minor addiction severity not branching into several areas of a patient's life. Also, the results cannot be generalised to other populations with more severe AUD profiles (e.g. an in-patient setting). Despite these limitations, this study adds to the evidence that childcare responsibilities need not be an obstacle to achieving favourable treatment outcomes for parents with AUD.

In conclusion, patients with childcare responsibilities have a less severe addiction profile at entry and a more coherent treatment course and better drinking-related outcomes compared with patients without similar responsibility when treated in conventional, evidence-based pharmacological and psychosocial out-patient treatment. This knowledge is crucial to guide clinical practice, effectuate interventions and inform social authorities. As a first line of treatment for patients with AUD living with children, healthcare and social care professionals may consider evidence-based out-patient treatment, hereby improving the family function and the welfare of the children. However, a thorough assessment of the degree of addiction severity and psychosocial impairment is needed, as these findings may not be generalisable to patients with AUD with more pronounced psychosocial impairment. In these cases, more complex integrative treatment, including more support and treatment for the diagnosed parent as well as provision of support for the children and other family members, may be needed. Additional studies should replicate the current findings in both out-patient and in-patient settings, and should investigate how treatment targeting the parents influences children's welfare.

\footnotetext{
Angelina Isabella Mellentin, PhD, Unit of Clinical Alcohol Research, Department of Clinical Research, University of Southern Denmark and Unit for Psychiatric Research, institute of Regional Health Services Research, University of Southern Denmark and, Institute of Regi, Unive Clinical Psychologist, Psychiatric Hospital, Odense University Hospital, Denmark; Annette Elkjær Ellermann, Clinical Psychologist, Psychiatric Hospital, Odense University Hospital, Denmark; Bent Nielsen, MD, PhD, Professor, Unit of Clinical Alcoho Research, Department of Clinical Research, University of Southern Denmark and Psychiatrist, Psychiatric Hospital, Odense University Hospital, Denmark; Anna Mejldal Statistician, Unit of Clinical Alcohol Research, Department of Clinical Research, University of Southern Denmark, Denmark: Sören Möller, Statistician, Professor, Odense Patient Data Explorative Network, Department of Clinical Research, University of Southern Denmark, Denmark; Anette Søgaard Nielsen, PhD, Associate Professor, Research Director, Unit of Clinical Alcohol Research, Department of Clinical Research, University of Southern Denmark, Denmark
}

Correspondence: Angelina Isabella Mellentin, Unit of Psychiatric Research, Department of Psychiatry, Odense University Hospital, Søndre Boulevard 29, DK-5000 Odense C, Denmark. Email: amellentin@health.sdu.dk

First received 26 Mar 2018, final revision 8 Oct 2018, accepted 8 Oct 2018

\section{References}

1 Grant BF. Estimates of US children exposed to alcohol abuse and dependence in the family. Am J Public Health 2000; 90(1): 112-5.

2 Yoon G, Westermeyer J, Kuskowski MA, Nesheim L. Impact of the number of parents with alcohol use disorder on alcohol use disorder in offspring: a population-based study. J Clin Psychiatry 2013; 74(8): 795-801.
3 Manning V, Best DW, Faulkner N, Titherington E. New estimates of the number of children living with substance misusing parents: results from UK national household surveys. BMC Public Health 2009; 9(1): 377.

4 Grekin ER, Brennan PA, Hammen C. Parental alcohol use disorders and child delinquency: the mediating effects of executive functioning and chronic family stress. J Stud Alcohol 2005; 66(1): 14-22.

5 Hussong AM, Bauer DJ, Huang W, Chassin L, Sher KJ, Zucker RA. Characterizing the life stressors of children of alcoholic parents. J Fam Psychol 2008; 22(6): 819-32.

6 El-Sheikh M, Flanagan E. Parental problem drinking and children's adjustment: family conflict and parental depression as mediators and moderators of risk. $J$ Abnorm Child Psychol 2001; 29(5): 417-32.

7 Rounsaville D, O'Farrell TJ, Andreas JB, Murphy CM, Murphy MM. Children's exposure to parental conflict after father's treatment for alcoholism. Addict Behav 2014; 39(7): 1168-71.

8 Christoffersen MN, Soothill K. The long-term consequences of parental alcohol abuse: a cohort study of children in Denmark. J Subst Abuse Treat 2003; 25(2): 107-16.

9 Woodin EM, Caldeira V, Sotskova A, Galaugher T, Lu M. Harmful alcohol use as a predictor of intimate partner violence during the transition to parenthood: interdependent and interactive effects. Addict Behav 2014; 39(12): 1890-7.

10 Dube SR, Anda RF, Felitti VJ, Croft JB, Edwards VJ, Giles WH. Growing up with parental alcohol abuse: exposure to childhood abuse, neglect, and household dysfunction. Child Abuse Negl 2001; 25(12): 1627-40.

11 Freisthler B, Gruenewald PJ. Where the individual meets the ecological: a study of parent drinking patterns, alcohol outlets, and child physical abuse. Alcohol Clin Exp Res 2013; 37(6): 993-1000.

12 Walsh $\mathrm{C}$, MacMillan $\mathrm{HL}$, Jamieson $\mathrm{E}$. The relationship between parental substance abuse and child maltreatment: findings from the Ontario Health Supplement. Child Abuse Negl 2003; 27(12): 1409-25.

13 Hill SY, Tessner KD, McDermott MD. Psychopathology in offspring from families of alcohol dependent female probands: a prospective study. J Psychiatr Res 2011; 45(3): 285-94.

14 Lieb R, Merikangas KR, Höfler M, Pfister H, Isensee B, Wittchen H-U. Parental alcohol use disorders and alcohol use and disorders in offspring: a community study. Psychol Med 2002; 32(01): 63-78.

15 Mellentin Al, Brink M, Andersen L, Erlangsen A, Stenager E, Bjerregaard LB, et al. The risk of offspring developing substance use disorders when exposed to one versus two parent (s) with alcohol use disorder: a nationwide, register-based cohort study. J Psychiatr Res 2016; 80: 52-8.

16 Sørensen HJ, Manzardo AM, Knop J, Penick EC, Madarasz W, Nickel EJ, et al. The contribution of parental alcohol use disorders and other psychiatric illness to the risk of alcohol use disorders in the offspring. Alcohol Clin Exp Res 2011; 35(7): 1315-20.

17 Jones R, Everson-Hock E, Papaioannou D, Guillaume L, Goyder E, Chilcott J, et al. Factors associated with outcomes for looked-after children and young people: a correlates review of the literature. Child Care Health Dev 2011; 37(5): 613-22.

18 Rutter M. Children in substitute care: some conceptual considerations and research implications. Child Youth Serv Rev 2000; 22(9-10): 685-703.

19 Tarren-Sweeney M. Retrospective and concurrent predictors of the mental health of children in care. Child Youth Serv Rev 2008; 30(1): 1-25.

20 Patterson ML, Moniruzzaman A, Somers JM. History of foster care among homeless adults with mental illness in Vancouver, British Columbia: a precursor to trajectories of risk. BMC Psychiatry 2015; 15(1): 32.

21 Dworsky A, Courtney ME. Homelessness and the transition from foster care to adulthood. Child Welfare 2009; 88(4): 23.

22 Fowler PJ, Toro PA, Miles BW. Pathways to and from homelessness and associated psychosocial outcomes among adolescents leaving the foster care system. Am J Public Health 2009; 99(8): 1453-8.

23 Meier PS, Donmall MC, McElduff P. Characteristics of drug users who do or do not have care of their children. Addiction 2004; 99(8): 955-61.

24 Swift W, Copeland J, Hall W. Characteristics of women with alcohol and other drug problems: findings of an Australian national survey. Addiction 1996; 91 (8): $1141-50$

25 Koski-Jannes A. The role of children in the recovery of alcoholic clients. Contemp Drug Probs 1991; 18: 629.

26 Stewart D, Gossop M, Trakada K. Drug dependent parents: childcare responsibilities, involvement with treatment services, and treatment outcomes. Addict Behav 2007; 32(8): 1657-68

27 Comiskey CM. A 3 year national longitudinal study comparing drug treatment outcomes for opioid users with and without children in their custodial care at intake. J Subst Abuse Treat 2013; 44(1): 90-6.

28 Comiskey CM, Hyland J, Hyland P. Parenthood, child care, and heroin use: outcomes after three years. Subst Use Misuse 2016; 51(12): 1600-9. 
29 World Health Organization. The ICD-10 Classification of Mental and Behavioural Disorders: Clinical Descriptions and Diagnostic Guidelines. World Health Organization, 1992.

30 World Health Organization. The ICD-10 Classification of Mental and Behavioural Disorders: Diagnostic Criteria for Research. World Health Organization, 1993.

31 McLellan AT, Kushner H, Metzger D, Peters R, Smith I, Grissom G, et al. The fifth edition of the Addiction Severity Index. J Subst Abuse Treat 1992; 9(3): 199-213.

32 Blacken P, Hendriks V, Pozzi G, Tempesta E, Hartgers C, Koeter M, et al. European Addiction Severity Index (EuropASI) Manual. European Monitoring Centre for Drugs and Drug Addiction, 2010 (http://www.emcdda.europa.eu/ html.cfm/index3647EN.html).

33 Kokkevi A Hartgers C. EuropASl: European adaptation of a multidimensional assessment instrument for drug and alcohol dependence. Eur Addict Res 1995; 1(4): 208-10.

34 McGahan PL, Griffith JA, Parente R, McLellann AT. Composite Scores Manual. Treatment Research Institute, 1986

35 Seaman SR, White IR. Review of inverse probability weighting for dealing with missing data. Stat Methods Med Res 2013; 22(3): 278-95.

36 Cox G, Comiskey C. Characteristics of opiate users presenting for a new treatment episode: baseline data from the national drug treatment outcome study in Ireland (ROSIE). Drugs 2007; 14(3): 217-30.

37 Conners NA, Grant A, Crone CC, Whiteside-Mansell L. Substance abuse treatment for mothers: treatment outcomes and the impact of length of stay. $J$ Subst Abuse Treat 2006; 31(4): 447-56.

38 Stevens SJ, Patton T. Residential treatment for drug addicted women and their children: effective treatment strategies. Drugs Soc 1998; 13(1-2): 235-49.

39 Szuster RR, Rich LL, Chung A, Bisconer SW. Treatment retention in women's residential chemical dependency treatment: the effect of admission with children. Subst Use Misuse 1996; 31(8): 1001-13.
40 Hughes PH, Coletti SD, Neri RL, Urmann CF, Stahl S, Sicilian DM, et al. Retaining cocaine-abusing women in a therapeutic community: the effect of a child live-in program. Am J Public Health 1995; 85(8 Pt 1): 1149-52.

41 Sanvisens A, Zuluaga P, Fuster D, Rivas I, Tor J, Marcos M, et al. Long-term mortality of patients with an alcohol-related Wernicke-Korsakoff syndrome. Alcohol Alcohol 2017; 52(4): 466-71.

42 Schuckit MA. Alcohol-use disorders. Lancet 2009; 373(9662): 492-501.

43 Cargiulo T. Understanding the health impact of alcohol dependence. Am J Health Syst Pharm 2007; 64(5 Suppl 3): S5-11.

44 Niccols A, Milligan K, Smith A, Sword W, Thabane L, Henderson J. Integrated programs for mothers with substance abuse issues and their children: a systematic review of studies reporting on child outcomes. Child Abuse Negl 2012; 36(4): 308-22.

45 Neger EN, Prinz RJ. Interventions to address parenting and parental substance abuse: conceptual and methodological considerations. Clin Psychol Rev 2015; 39: 71-82.

46 Milligan K, Niccols A, Sword W, Thabane L, Henderson J, Smith A, et al. Materna substance use and integrated treatment programs for women with substance abuse issues and their children: a meta-analysis. Subst Abuse Treat Prev Policy 2010; 5(1): 21.

47 Wilke DJ, Kamata A, Cash SJ, Modeling treatment motivation in substanceabusing women with children. Child Abuse Neg/ 2005; 29(11): 1313-23.

48 Finkelstein $\mathrm{N}$. Treatment issues for alcohol-and drug-dependent pregnant and parenting women. Health Soc Work 1994; 19(1): 7-15.

49 Adamson SJ, Sellman JD, Frampton CM. Patient predictors of alcohol treatment outcome: a systematic review. J Subst Abuse Treat 2009; 36(1): 75-86. 